\title{
Histomorphometric and Ultrastructural Evaluation of Long-Term Alpha Lipoic Acid and Vitamin B12 Use After Experimental Sciatic Nerve Injury in Rats
}

\author{
Murat ARIKAN ${ }^{1}$, Guray TOGRAL ${ }^{1}$, Askin Esen HASTURK ${ }^{2}$, Bahriye HORASANLI ${ }^{3}$, Fatma HELVACIOGLU ${ }^{4}$, \\ Atilla DAGDEVIREN ${ }^{4}$, Mustafa Agah TEKINDAL ${ }^{5}$, Murat PARPUCU ${ }^{6}$
}

\author{
${ }^{1}$ Oncology Training and Research Hospital, Department of Orthopedics and Traumatology, Ankara, Turkey \\ ${ }^{2}$ Oncology Training and Research Hospital, Department of Neurosurgery, Ankara, Turkey \\ ${ }^{3}$ Baskent University Medical School, Department of Neurology, Konya, Turkey \\ ${ }^{4}$ Baskent University Medical School, Department of Histology and Embryology, Ankara, Turkey \\ ${ }^{5}$ Baskent University Medical School, Department of Biostatistics, Ankara, Turkey \\ ${ }^{6}$ Oncology Training and Research Hospital, Department of Anesthesiology, Ankara, Turkey
}

\section{ABSTRACT}

AIM: To analyze the therapeutic effects of long-term alpha lipoic acid (A-LA) and vitamin B12 use via histomorphometric methods and electron microscopy in the transected sciatic nerves of rats.

MATERIAL and METHODS: Forty rats were randomized into five groups ( $\mathrm{n}=8 / \mathrm{group})$. In group I, $1 \mathrm{~cm}$ segment of sciatic nerve was resected without any other intervention. In group II (sham), following right sciatic nerve transection, primary epineurial anastomosis was performed by placing the edges of the nerve end-to-end. In group III (saline), after right sciatic nerve transection, the ends of the nerves were brought together and closed after application of intraperitoneal physiologic saline. In group IV, $2 \mathrm{mg} / \mathrm{kg}$ of alpha lipoic acid and in group V, $2 \mathrm{mg} / \mathrm{kg}$ of vitamin B12 was administered intraperitoneally before surgical intervention.

RESULTS: Histomorphometric and electron microscopic analyses revealed that vitamin B12 did not prevent structural changes, abnormal myelination and g-ratio deviations regarding the functional aspects of the sciatic nerve. Alpha lipoic acid was more effective in restructuring the histomorphometric and structural aspects of the nerve with more myelinated fibers with optimal values (0.55-0.68) than vitamin B12 groups, in which the number of myelinated nerve fibers significantly decreased at optimal intervals (0.55-0.68).

CONCLUSION: A-LA administration following peripheral nerve transection injury is more effective in promoting nerve healing regarding the structural aspects of the sciatic nerve compared to vitamin B12 and also myelination of nerve fibers by increasing g-values.

KEYWORDS: Alpha lipoic acid, Vitamin B12, g ratio, Sciatic nerve, Histomorphometry

\section{INTRODUCTION}

S ciatic nerve transection injuries are common following nerve crushes in accidents, iatrogenic nerve cutting in surgical procedures, fractures, skin lacerations and achieving a full recovery is still a major challenge because of complex pathophysiological mechanisms. These injuries affect primarily younger patients and functional recovery rarely returns to the preinjury level (6). Sciatic nerve injuries with neurological deficits have a variable recovery course, and if the sensory and motor deficits do not resolve spontaneously, surgical interventions are necessary. In a recent study, nerve 
lesions were found to be associated with a surgical procedure in $94 \%$ of patients, and approximately $25 \%$ of sciatic nerve injuries are secondary to medical interventions (1). The gold standard treatment for peripheral nerve transection is anatomically rejoining the axon and suturing the nerve by a microsurgical technique (14). Experimental studies show that the regeneration of nerve fibers are most favorable in the first days and weeks following injury so the decision whether an operative reconstruction or conservative therapy is needed will be judged according to the severity of structural damage following peripheral nerve injury (17).

New studies emphasize the effectiveness of the antioxidant effects of some medications like $\alpha$-lipoic acid (A-LA) on the crush injury of rat sciatic nerve after peripheral nerve injuries. Also some drugs, medications and food supplements like vitamin B12 are being trailed for possible regenerating effects on peripheral nerves after injury. Histomorphometric studies on the g-ratio are gaining popularity to evaluate the relationship between nerve conduction velocity and fiber morphology during peripheral nerve regeneration (4). These studies are supposed to give valuable data about the accelerating and ameliorating effects of these medications on peripheral nerve regeneration.

A-LA is an antioxidant that plays an important role as a cofactor in multiple mitochondrial reactions, is readily absorbed from the diet and easily crosses the blood brain barrier (7) and a powerful metabolic antioxidant with immunomodulatory effects that provide neuroprotection. In experimental studies, A-LA treatment in rodents has been shown to reduce the damage that occurs after ischemia-reperfusion injuries in the cerebral cortex, heart and peripheral nerve (21). The therapeutic effects of A-LA on transient cerebral ischemia, Alzheimer's disease and traumatic brain injury have been reported previously (8). Vitamin B12 also plays an important role in attenuating degenerative processes in the nervous system, and severe damage occurs in the spinal cord and central nervous system (CNS) in the presence of vitamin B12 deficiency (20). Vitamin B12 deficiency causes a wide range of hematological, gastrointestinal, psychiatric and neurological disorders, and neuropsychiatric symptoms may present as myelopathy, neuropathy, dementia and, less often, optic nerve atrophy (3). Also this vitamin attenuates degenerative processes in the nervous system and promotes the regeneration of myelinated nerve fibers and the proliferation of Schwann cells (5).

In this experimental study, the therapeutic effects of longterm alpha lipoic acid and vitamin B12 treatment using histomorphometric analysis and electron microscopy in the transected sciatic nerves of rats are analyzed.

\section{MATERIAL and METHODS}

Animal care and all experiments were in accordance with the European Communities Council Directive of November 24, 1986 (86/609/EEC) on the protection of animals for experimental use. All experimental procedures used in this research were approved by the ethics committee of the Ministry of Health Ankara Education and Research Hospital. In this study, Sprague-Dawley male rats weighing approximately 250-300 grams were analyzed with 8 rats divided into 5 groups for a total of 40 rats.

\section{Surgical Procedure}

All of the rats were anesthetized with a combination of 10 $\mathrm{mg} / \mathrm{kg}$ xylazine $\mathrm{HCl}$ ((Rompun 2\%; Bayer HealthCare AG, Germany) and $80 \mathrm{mg} / \mathrm{kg}$ ketamine $\mathrm{HCl}$ (Ketasol 10\% inf; $10 \mathrm{ml}$ vial; Richter Pharma) at a dose of $40-50 \mathrm{mg} / \mathrm{kg}$ intraperitoneally with the body temperature maintained at $37-58^{\circ} \mathrm{C}$. Overall, 5 rats were kept in a cage specifically designed for their daily living intra-and post-operatively, and we attached markers with a unique number to their ears to distinguish between the animals. The rats were allowed mobilization in the cage and sufficient food and water. Appropriate antibiotics and analgesics were given before the surgical intervention. Under prone position, the rat extremities were fixed on the operation table, and the operation area was elevated by placing a $1 \mathrm{~cm}-$ thick roll of gauze bandage underneath the table, and the area was cleaned using iodine (Glividon®), Bikar llac San, Istanbul, Turkey) solution. A $3 \mathrm{~cm}$ posterior longitudinal skin incision was made from the right gluteal region through the posterior femoral region. Fascia that surrounds the junction line of the gluteus superficialis and the biceps femoris and its surface were explored with blunt dissection, and the sciatic nerve was exposed. The sciatic nerve was isolated from the surrounding tissues by dissecting the superficial membranous structures on the unifascicular area from the sciatic foramen to the point at which the tibial and peroneal branches were separated. A regular full-fold nerve incision was performed using microscissors by slightly elevating $(10 \mathrm{~mm})$ the sciatic nerve from sciatic foramen with a root hook. Using the epineurial nerve repair technique, proximal and distal rudimentary nerves were sutured from two different points separated with an angle of $180^{\circ}$ using an 8-0 polypropylene suture (Prolene, Ethicon ${ }^{\circledR}$ Ltd, Somerville NJ, USA), and primary anastomosis was created. To prevent reinnervation, the proximal stump of the nerve was buried under the gluteus maximus musculature. The skin was then closed via suture using a 4-0 polypropylene (Prolene, Ethicon @Ltd, Somerville NJ, USA) suture. All rats recovered spontaneously from anesthesia, and they were housed in groups of 5 in each cage after surgical intervention and given free access to food and water.

\section{Experimental Groups}

Rats were randomly assigned to the following five groups:

Group I (Control) $(\mathrm{n}=8): 1 \mathrm{~cm}$ of sciatic nerve segment was resected and taken out without any other intervention. The rats were maintained for 12 weeks before being sacrificed.

Group II (Sham) ( $n=8)$ : After right sciatic nerve transection, primary epineurial anastomosis was performed by bringing the edges of the nerve end-to-end. The rats were maintained for 12 weeks before being sacrificed.

Group III (Saline) ( $n=8)$ : After right sciatic nerve transection, the ends of the nerves were brought together and closed after the application of the intraperitoneal physiological saline. The medical treatment continued for 12 weeks before the animals were sacrificed. 
Group IV (Alpha lipoic acid) ( $n=8)$ : After right sciatic nerve transection, the ends of the nerve were brought together and sutured with primary epineurial anastomosis. Additionally, 2 $\mathrm{mg} / \mathrm{kg}$ of alpha lipoic acid was administered intraperitoneally to the rats before surgical intervention, and the medical treatment continued for 12 weeks before the animals were sacrificed.

Group V (Vitamin B12) ( $n=8)$ : After right sciatic nerve transection, the ends of the nerve were brought together and sutured with primary epineurial anastomosis. Additionally, 2 $\mathrm{mg} / \mathrm{kg}$ of vitamin B 12 was administered intraperitoneally to the rats before surgical intervention, and the medical treatment continued for 12 weeks before the animals were sacrificed.

All of the rats were sacrificed (Thiopental Sodium $150 \mathrm{mg} / \mathrm{kg} / \mathrm{IP}$ ) for macroscopic, histomorphometric and electron microscopic evaluation (axonal regeneration and reorganization) at the end of the 12-week period. The sciatic nerves were then excised en-bloc with the tissue surrounding the introduction and the segment that was repaired, and they were placed in $4 \%$ glutaraldehyde for histopathological examination.

\section{Macroscopic Evaluation}

Self-harming behaviors following the surgical procedure were monitored. Skin ulcerations, hemorrhagic foci and hematomas were observed on the right toes of several rats due to a sciatic nerve deficit. Spontaneous amputations of the toes due to self-mutilation were also noted in 2 rats. The skin incision was opened for macroscopic evaluations of the fascia, and the adhesion of the nerve to the surrounding muscular mass and the mobility and separation of the nerve from these structures were evaluated.

No signs of infection around the incision site were recorded in any rats during the exploration of the sciatic nerve. In all groups and in all rats, the anastomosis site was intact, and the proximal and distal edges of the nerve were not separated from each other with traction. At the anastomosis site, nerve tissue was dissected, and the peripheral soft tissue was removed with sharp dissection. In total, $1 \mathrm{~mm}$ from distal and $1 \mathrm{~mm}$ from the proximal edge of the nerve including the sutures was transected with a microknife and removed for histological analysis.

\section{Histomorphometric Analysis}

Histomorphometric evaluation was conducted using the semi-thin sections at $\times 1000$ magnification, and the g-ratio for the myelinated axons was assessed. Randomly selected fields of transverse sections of sciatic nerves from rats for each group (150 fibers per group) were analyzed. The structural and functional aspects of the sciatic nerve optimal g-ratio from 0.55 to 0.68 were considered (4). The g-ratio was calculated by dividing the inner axonal diameter by the outer fiber diameter, and the results were stratified in ranges of 0.3$0.49,0.5-0,54,0.55-0.68$, and 0.69-0.8. Quantifications were performed using, a program written specifically to calculate the g-ratio in peripheral nerve research.

\section{Electron Microscopic Evaluation}

Tissues from all the groups were fixed in phosphate buffered solution $(\mathrm{Ph}=7.3)$ containing $2.5 \%$ glutaraldehyde (SigmaAldrich Co.) for $2 \mathrm{~h}$ at room temperature, post-fixed in $1 \%$ osmium tetraoxide (Sigma-Aldrich Co.) and dehydrated in a series of graded alcohols $(50,60,70,80,90$ and $100 \%$ ethanol). After passing through propylene oxide (SigmaAldrich Co.), the specimens were embedded in Araldite CY 212 (Ciba-Geigy), (2-dodecen-1-yl) succinic anhydride (Sigma-Aldrich Co.), benzyldimethylamine (Poly Sciences Inc.) and dibutylphtalate (Sigma-Aldrich Co.). The semi-thin sections were stained with toluidine blue (Sigma-Aldrich Co.) and examined with a photomicroscope (DM 500 Leica, Germany). After the selection of appropriate specimens, thin sections were cut, stained with uranyl acetate (ProSciTech) and lead citrate (Sigma-Aldrich Co.), and examined via an electron microscope by two blinded histologists (Leo $906 \mathrm{E}$ Carl Zeiss, Germany).

\section{Statistical Analysis}

The results are specified as numbers ( $n$ ) and percentages (p). Categorical data were analyzed with Fisher's Exact Test and the Chi Squared Test. In the case where the expected frequencies were lower than 5 , the Monte Carlo Simulation Method was chosen. $p<0.05$ level was considered statistically significant. The data were analyzed using the SPSS Statistics software program (Version 17, Chicago IL, USA).

\section{RESULTS}

\section{Histomorphometric Findings}

In this evaluation, myelinized nerve fibers that were regularly arranged in the control groups had an optimal g-ratio at a percentage of $83.3 \%$; however, in the trauma with saline and trauma with vitamin B12 groups, the number of myelinated nerve fibers significantly decreased at optimal intervals $(0.55-0.68)$, in the group treated with alpha lipoic acid, the percentage of myelinated fibers with optimal values $(0.55-$ $0.68)$ was similar to the results of the control group $(p<0.01)$ (Figure 1).

\section{Structural Findings}

In the control group, the sciatic nerve sections of the myelin sheath, axons and epineurium were found to be normal under both small and large magnification. Under the epineurium near a thin layer of soft connective tissue layer, myelinated nerve fibers with different diameters and blood vessels in the endoneurial compartment were identified. Damage observed in a few large diameter myelinated nerve fibers was neglected because the damage looked typical and common (Figure $2 \mathrm{~A}, \mathrm{~B})$. Electron microscopy examination of the control group revealed small and large diameter axons with myelin, SchmidtLantermann clefts in the myelin sheath, axons without myelin surrounded with Schwann nerves and collagen nerve fibers arranged in a regular fashion in the connective tissue (Figure 2C). 
In the sham trauma group, the non-magnified and magnified images showed myelinated nerve fiber groups phagocytosed by macrophages (Figure $3 \mathrm{~A}$ ) under the epineurium and in the endoneurial compartment and Wallerian-like degeneration in the small and large myelinated nerves with definitive structural damage, unlike the control group. Additionally, blood vessels were observed in the endoneurial compartment (Figure $3 \mathrm{~A}, \mathrm{~B})$. In the sham trauma group, unlike the control group, myelinated axon anomalies were detected as a composition of myelin sheaths of different diameters inside the axon of a myelinated nerve fiber as described by Saito and colleagues (19) in dystroglycan knock out mice. Interestingly, more than one myelinated nerve fiber was observed in Schwann cell cytoplasm. Two different myelin sheath structures were identified in the large myelinated nerve fiber inside a polyaxonal Schwann cell. Furthermore, in this group, myelin sheath degeneration had a granular appearance, and nerve fibers with grossly thickened myelin sheaths were observed (Figure 3C). In distinct field examination, the myelinated nerve fibers had normal structures, and two large-diameter myelinated nerve fibers in the cell cytoplasm of Schwann cells and degenerative e-dense deposits were observed. In one of the large-diameter fibers, the axon was completely covered with a myelin sheath, and in the other fiber, myelin sheath degeneration with a granular appearance was recorded. In several Schwann cells, the number of organelles was increased. Regression in the axons in several sections and e-dense depositions in the axoplasms were recorded, and the myelin sheath had irregular thickness in some sections. Normal structures were recorded in several parts of the nerve fibers; however, some nerve fibers without myelin showed swelling. Unlike the control group, an increase in the intermediate connective tissue and relative augmentation of the collagen fibers was observed via electron microscopy (Figure 3D).

Similar findings were observed in the sham trauma and trauma-saline groups. In the semi-thin sections, while the large diameter fibers could not be distinguished, the small diameter fibers had a regular arrangement of the myelin in several sections. Additionally, the connective tissue under the epineurium was definitely augmented compared to the trauma group, capillary vessels were present, and blood vessels in the endoneurial compartment were recorded. This finding suggests that the angiogenesis mechanism was triggered after trauma (Figure 4A, B). Polyaxonal Schwann cells were observed in magnified and non magnified sections in hyperfine structures of both of these groups. Axonal degeneration of the myelinated nerve fibers was distinctive. In the low magnified sections, the organelles were increased in the cytoplasm of the Schwann nerves. Vacuole formation was present in the fibers in which the myelin sheath structure was preserved. The swelling in the nerve fibers without myelin was manifested in this trauma-saline group. Furthermore, the edema was less intense in the intermediate connective tissue compared to the trauma group, and the increase in swelling was recorded in the arrangement of collagen fibers (Figure $4 \mathrm{C}$ ). The collapse or penetration of the myelin sheath in the polyaxonal Schwann cells and inside the myelinated axon indicates that the Schwann cells failed to complete the myelination process. Unlike the control group, in the sham trauma and trauma saline groups were seen structural integrity can not be preserved. The most dramatic changes in the sham trauma and trauma saline groups were determined as polyaxonal Schwann cells and e dense deposits in large myelinated axons.

In evaluating the healing effect of vitamin B12 after traumatic injury via light microscopy, large diameter nerve myelinated nerve fibers were not examined because small diameter nerve fibers were dominant in the tissue. The presence of capillaries under the epineurium and the blood vessels in the endoneurial compartment was recorded in this group. In the magnified sections, the phagocytosed myelinated axons and the large diameter axons did not preserve their structural integrity; however, the small diameter nerve fibers

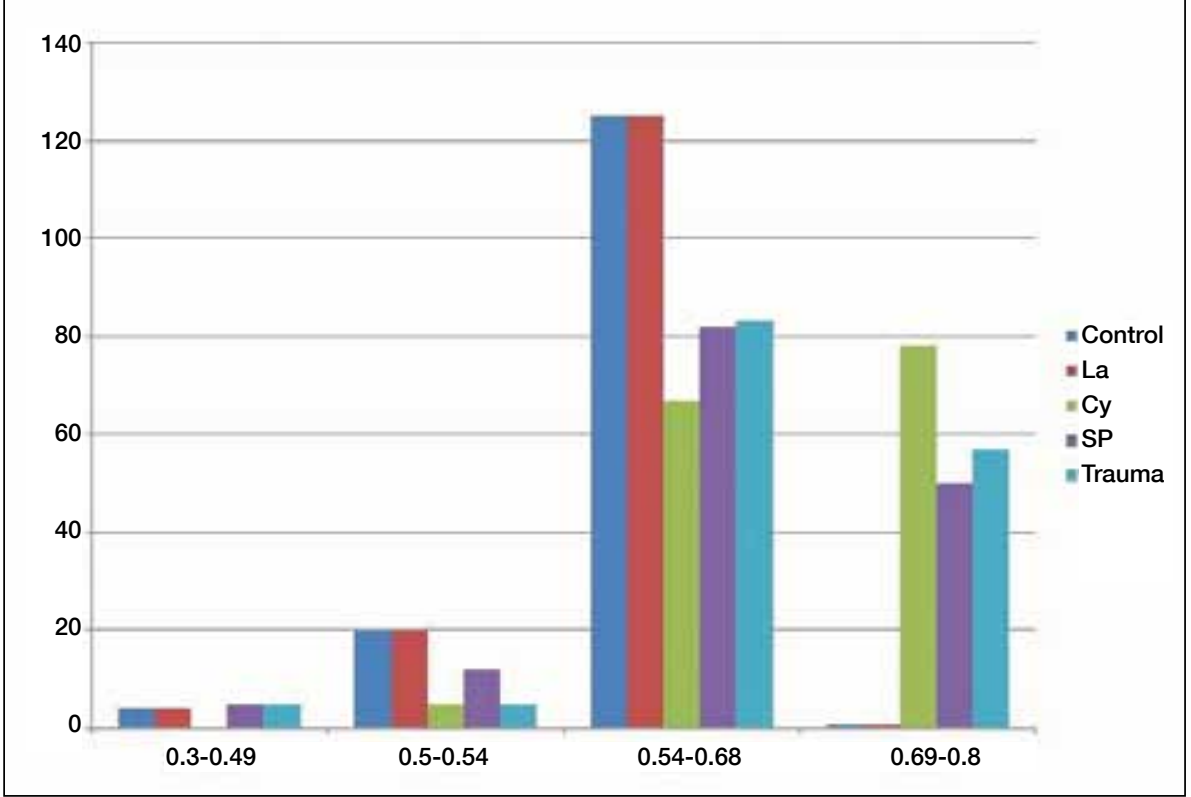

Figure 1: Categorical distribution of g-ratios among the analyzed groups. Statistically significant correlation between among the g-ratios and groups. ${ }^{* *} p<0.01$. La: Lipoic acid, Cy: vitamin B12, SP: Serum Physiologic. 

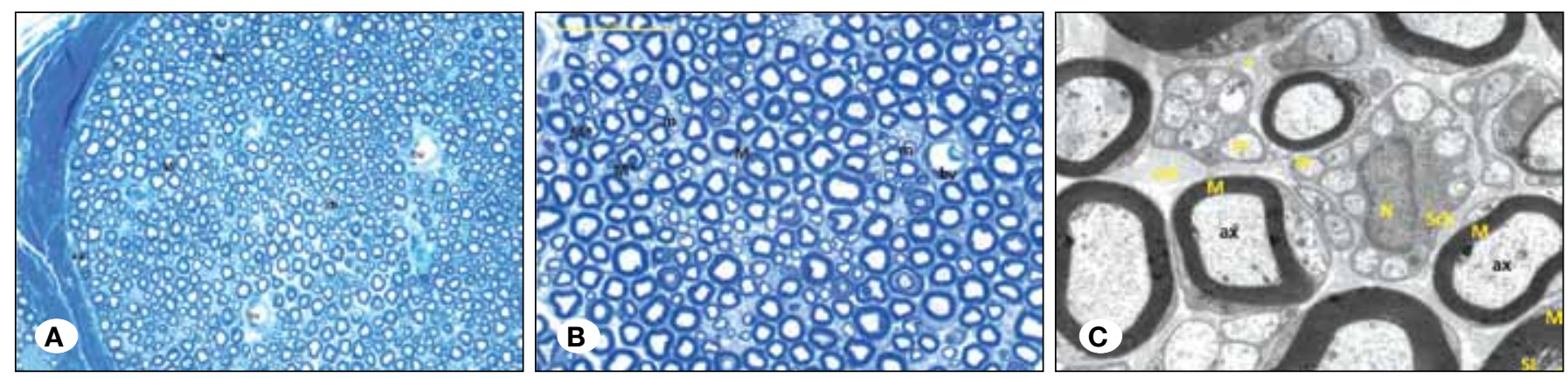

Figure 2: A, B) Control Group Semi-Thin Section; M: Large diameter nerve fiber with myelin, $M^{*}$ : Degenerated large diameter nerve root with, m: Small diameter nerve fiber with myelin, Bv: Blood vessels in endoneurial compartment, 2: Connective tissue under epineurium. (Toluidine Blue A x400- B x1000). C) Control Group Thin Section; M: Large diameter nerve fiber with myelin, m: Nerve fiber without myelin, ax: axon, SL: Schmidt-Lantermann cleft, Sch; Schwann cell, N: Schwann nerve nucleus, +: intermediate connective tissue, col; collagen bundles (uranyl acetate\& lead citrate $x$ 2750).

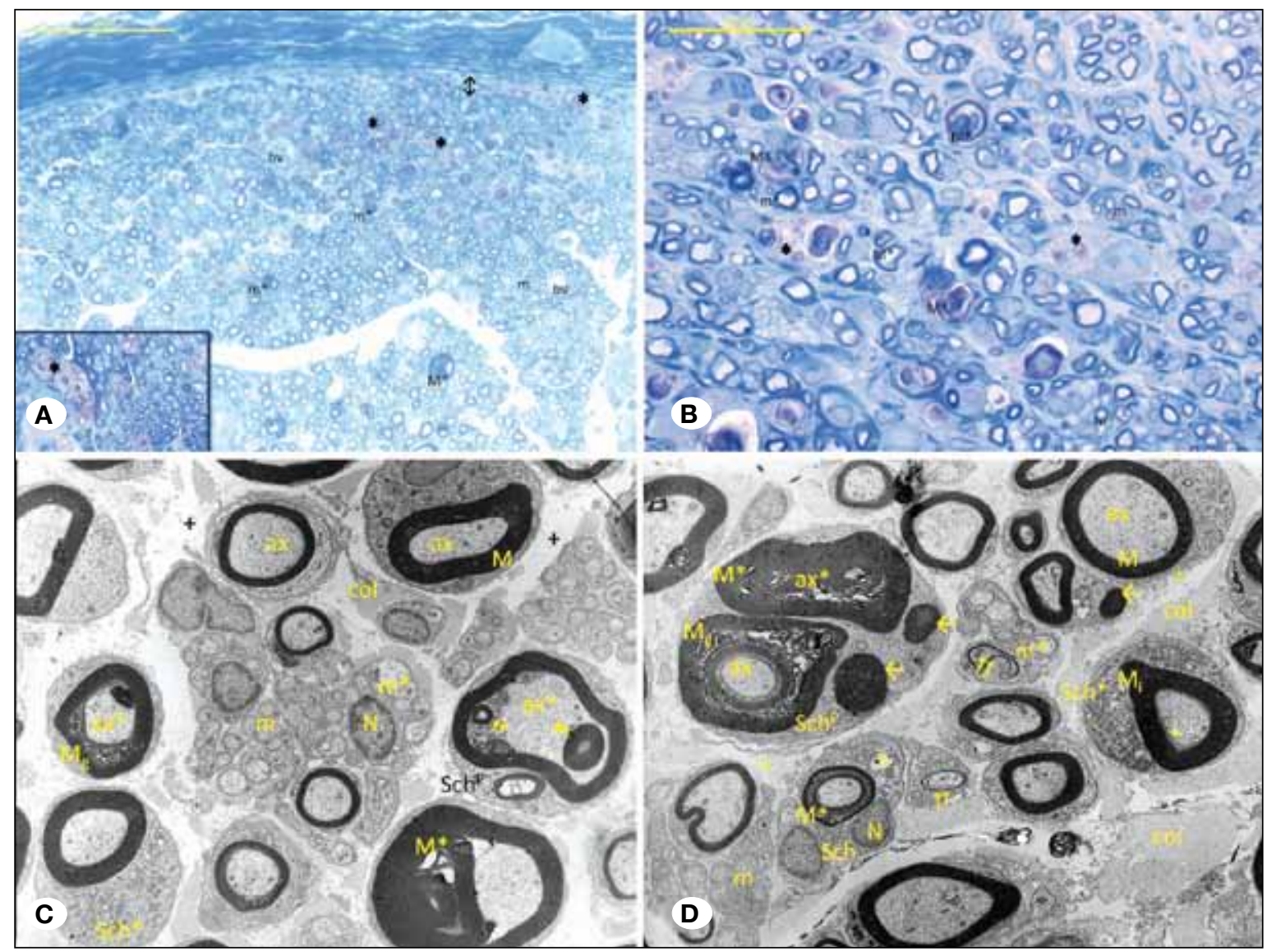

Figure 3: A, B) Trauma Group Semi-Thin Section; $M$ : Large diameter nerve fiber with myelin, $M_{\mathrm{g}}$ : Degenerative large diameter nerve fiber with, $\mathrm{m}$ : Small diameter nerve fiber with myelin, $\mathrm{m}^{*}$ : Degenerative small diameter nerve fiber with myelin, Bv: Blood vessels in endoneurial compartment, 2: Connective tissue under epineurium, B; Myelin sheath clusters phagosited by macrophages (inset) (Toluidine blue A x400- B x1000). C) Trauma Group Thin Section; M: Normal myelin sheath, $M_{\mathrm{g}}$ : Degenerated myelin sheath, m: normal nerve fiber without myelin, $\mathrm{m}^{*}$ : Degenerative small diameter nerve fiber without myelin, ax: Normal axon, ax*; Degenerative axon, 8; Different diameter myelin structure in the nerve fiber, Sch; Schwann cell, N: Schwann cell nucleus, Sch; Polyaxonal Schwann cell, Sch*; Schwann cells having intense organelles, +: edema in the intermediate connective tissue, col; collagen fibers (uranyl acetate\& lead citrate x2156). D) Trauma Group Thin Sections; $M$ : Myelin sheath in a normal arrangement, $M^{*}$ : Degenerative myelin sheath, $M_{i}$ : myelin sheath in irregular thickness, l; nerve fiber showing abnormal myelination, $\mathrm{m}$ : nerve fiber without myelin in normal structure, $\mathrm{m}^{\star}$ : Degenerative nerve fiber without myelin, ax: normal axon, ax*; axon showing degenerative changes, Sch; Schwann cell, N: Schwann cell nucleus, Sch; ; Polyaxonal Schwann cell, Sch*; Schwann cells having intense organelles, à: e- dense deposits in Schwann cells, v: vacuole,+: edema in the intermediate connective tissue, col; collagen fibers (uranyl acetate\& lead citrate x2750). 

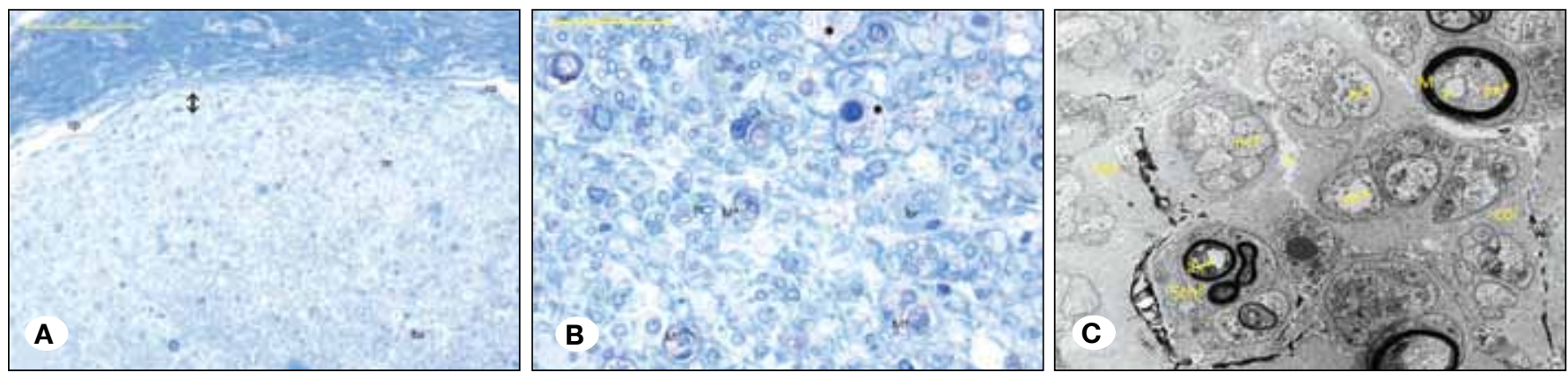

Figure 4: A, B) Trauma+ saline group semi-thin section; $M^{*}$ : Degenerative large diameter myelinated nerve fiber $m$ : small diameter nerve fiber with myelin, $\mathrm{m}^{*}$ : Degenerative small diameter myelinated nerve fiber, Bv: Blood vessel in endoneurial compartment, 2: connective tissue under epineurium, cp; Capillary, B; Myelin sheath clusters phagocytized by macrophages (Toluidine blue A x400- B x1000). C) Trauma+ Saline Group Thin Section; M: Myelin sheath in normal structure, $M^{\star}$ : Degenerated myelin sheath, $m^{\star}$ : Degenerated nerve fibers without myelin, ax*; Axon showing degenerative changes, Sch; Schwann nerve, Sch; Polyaxonal Schwann nerve, v: vacuole, +: Edema in intermediate connective tissue, col; Collagen fibers (uranyl acetate\& lead citrate X2156).

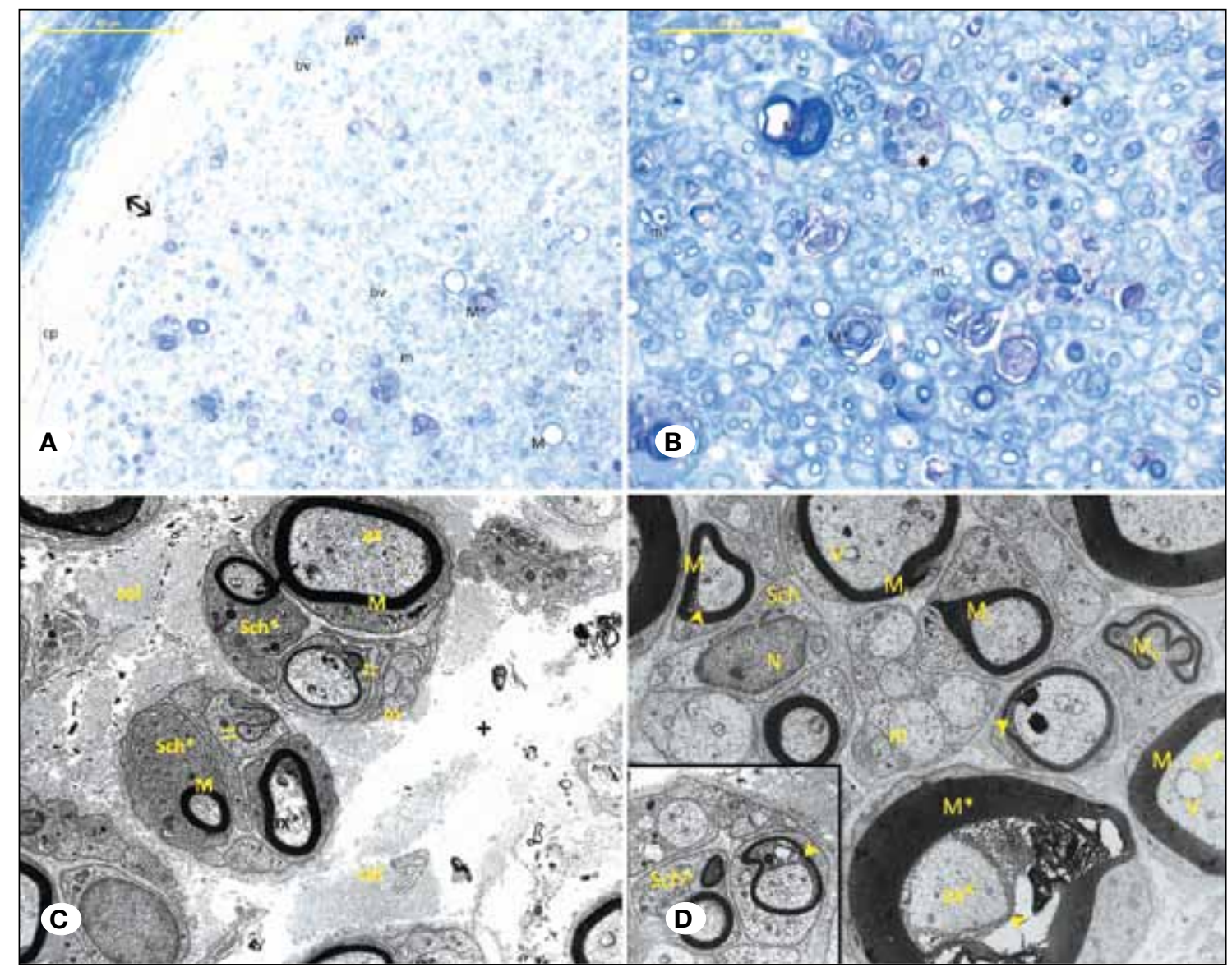

Figure 5: A,B) Trauma+vitB12 group semi-thin section; M: Large diameter nerve fiber with myelin $\mathrm{M}^{*}$ : Degenerative large diameter myelinated nerve fiber, $\mathrm{m}$ : Small diameter nerve fiber with myelin, $\mathrm{m}^{*}$ : Degenerative small diameter nerve fiber with myelin, Bv: Blood vessel in endoneurial compartment, 2: connective tissue under epineurium, $\mathrm{cp}$; Capillary, B; Myelin sheath clusters phagosited by macrophages (Toluidine blue A x400- B x1000). C) Trauma+vitB12 group thin section; M: Myelin sheath in normal structure myelin sheathl; Nerve fiber showing abnormal myelination, m: Nerve fiber without myelin in normal structure, ax: axon with normal configuration, ax*; Axon showing degenerative changes, Sch*; Schwann cells with intense organelles,+: edema in the intermediate connective tissue, col; collagen fibers (uranyl acetate\& lead citrate x3597). D) Trauma+vitB12 group thin section; M: Myelin sheath in normal configuration, $M^{*}$ : Degenerative myelin sheath, $M_{i}$ : myelin sheath with irregular thickness, $M_{0}$ : Myelinated nerve fiber showing ondulations, $m$ : Small diameter nerve fiber with a normal myelin configuration, ax*; Axon showing degenerative changes, $\dagger$; Areas of contact between the axon and myelin, Sch; Schwann cell, N: Schwann cell nucleus, v: vacuole,+: edema in the intermediate connective tissue, col; Collagen fibers $\mathrm{Sch}_{\mathrm{P}}$; Polyaxonal Schwann cell (inset) (uranyl acetate\& lead citrate x3597). 
preserved the myelin sheath structure in several fields, such as the trauma and trauma-saline groups (Figure 5A, B). In the thin sections, the edema was prominent in the intermediate connective tissue of the trauma and saline groups. While, the organization of the myelin sheath appeared normal in the small diameter fibers, nerve fibers with abnormal myelination were distinguished. Cytoplasmic organelles were very active in the Schwann cells (Figure 5C). In the same group, Schwann cells with prominent euchromatin and vacuole formation in the axoplasm were found. Prominent degeneration and the loss of integrity between the axon and myelin sheath in several areas were examined in large diameter nerve sheaths. However, swelling was prominent in the nerve fibers without myelin, and ondulations and thickness irregularities of several myelinated nerve fibers were observed. Polyaxonal Schwann cells were also examined in this group. In this group, the integrity of the sciatic nerve was preserved more than in the trauma groups; however, the edema remained high and the myelination process involved degenerative characteristics related to trauma origin (Figure 5D).

While determining the healing effects of A-LA on sciatic nerves after trauma, this treatment was determined to be more effective than the treatment of the previous group. In this group, in semi thin sections, magnified and small images revealed small and large diameter axons that preserve the natural architecture in addition to large diameter axons with myelin showing intense structural damage. In magnified images, large diameter fibers existed in several fields exhibiting myelin sheath structure as a thin layer. Distinctly different than the other groups (Group II, Group III, Group V), the connective tissue under the epineurium was decreased, the myelinated axon clusters were absent as they were phagocytosed by the macrophages and the axons were remodeling the myelin sheath in most of the areas (Figure 6A, B). In the cross-section of the tissue, the myelinated axons preserved their natural structure, while the myelinated nerve fibers showed abnormal myelination and minimal degeneration (Figure 6C). As shown by electron microscopy, myelin degeneration in several large diameter fibers occurred and disrupted the contacts between the axons and myelin. Additionally, the edema located in the intermediate connective tissue was reduced compared to other groups (Group II Group III, Group V). Polyaxonal Schwann nerves and Schwann nerves possessed euchromatic nuclei and rich organelles in this group, and e-deposits in the myelinated axons of these cells were observed. Although the fibroblasts had active endoplasmic reticulum in the intermediate connective tissue, the collagen fiber arrangements were apparently normal compared to the other groups that underwent trauma (Figure 6D). Unlike the control group, in the sham trauma and trauma saline groups were seen structural integrity can not be maintained. The most dramatic changes in the sham trauma and trauma saline groups were determined as polyaxonal Schwann cells and e dense deposits in large myelinated axons.

Briefly, vitamin B12 was found to be inadequate in avoiding the structural changes after trauma such as edema, myelin sheath degeneration, polyaxonal Schwann cells, e-dense depositions in Schwann cell cytoplasm and abnormal myelination and g-ratio deviation considering functional aspects. However, alpha lipoic acid was found to be more effective in the restructuring period of both the functional and structural aspects compared to vitamin B12.

\section{DISCUSSION}

Neural tissue repair and regeneration strategies have become increasingly popular because they directly affect patient quality of life. Functional healing after the repair of transected peripheral nerve injury is still poor despite recent advances in microsurgical interventions (2). While axons in peripheral nerves regenerate well, the axons present in the CNS regenerate poorly, which may be due to the inhibitory molecules of the CNS and a non-regenerative response to injury. Neural regeneration after injury is a very complex process, and Schwann cells play a critical role in the regeneration process (12). Regeneration process may take several months in peripheral nervous system (PNS). This process begins with the disintegration of axon. Then axons Schwann cell contact is lost. This event indicates the self-destruction of the myelin sheath. Macrophages begin to eliminate residues of myelin sheaths and migrate to injured area. The destruction and removal of waste products in the damaged area provides a healthy way to occur in axon regeneration. Injury to a peripheral nerve is followed by a cascade of pathophysiological events including the disorganization of the blood-nerve barrier, the recruitment of circulating macrophages, the proliferation of Schwann cells, raised cytokine production, rearrangement of endoneurial place, and the production of extra cellular components (18). These pathologic cascades contribute to the degeneration of motor neurons and loss of Schwann cells due to the absence of a suitable environment resulting in delayed nerve regeneration or irreversible neuronal damage.

Peripheral nerve regeneration necessitates interactions among multiple cells, neurotrophic factors and extracellular matrix proteins $(11,15)$. Axonal regeneration that results in axonal sprouting, growth and reinnervation is the most important part of this process.

Functional recovery necessitates axonal regeneration, remyelination and reinnervation of the sensory receptors, muscle end plates, or both (22). No definitive treatment for peripheral nerve injuries exists but early surgical intervention with end to end anastomosis is still the gold treatment modality in the first step of treatment (18). Experimentally, many medications have been used in rat crush injury models in order to speed up nerve regeneration and good neurologic recovery such as nonsteroidal anti-inflammatory drugs, steroids, low dose radiotherapy, and vitamins (23).

In the rat sciatic model, ultrastructural examination, electrophysiological studies and sciatic functional index assessments are the most popular methods to evaluate neural regeneration. Walking track analysis is also an important method that can test functional recovery after sciatic nerve transection injuries but in this study, the comparison of the myelination ratios of two contemporary drugs vitamin B12 and A-LA after experimentally transected sciatic nerve is discussed in regards to histomorphometric and electron microscopic evaluation. 


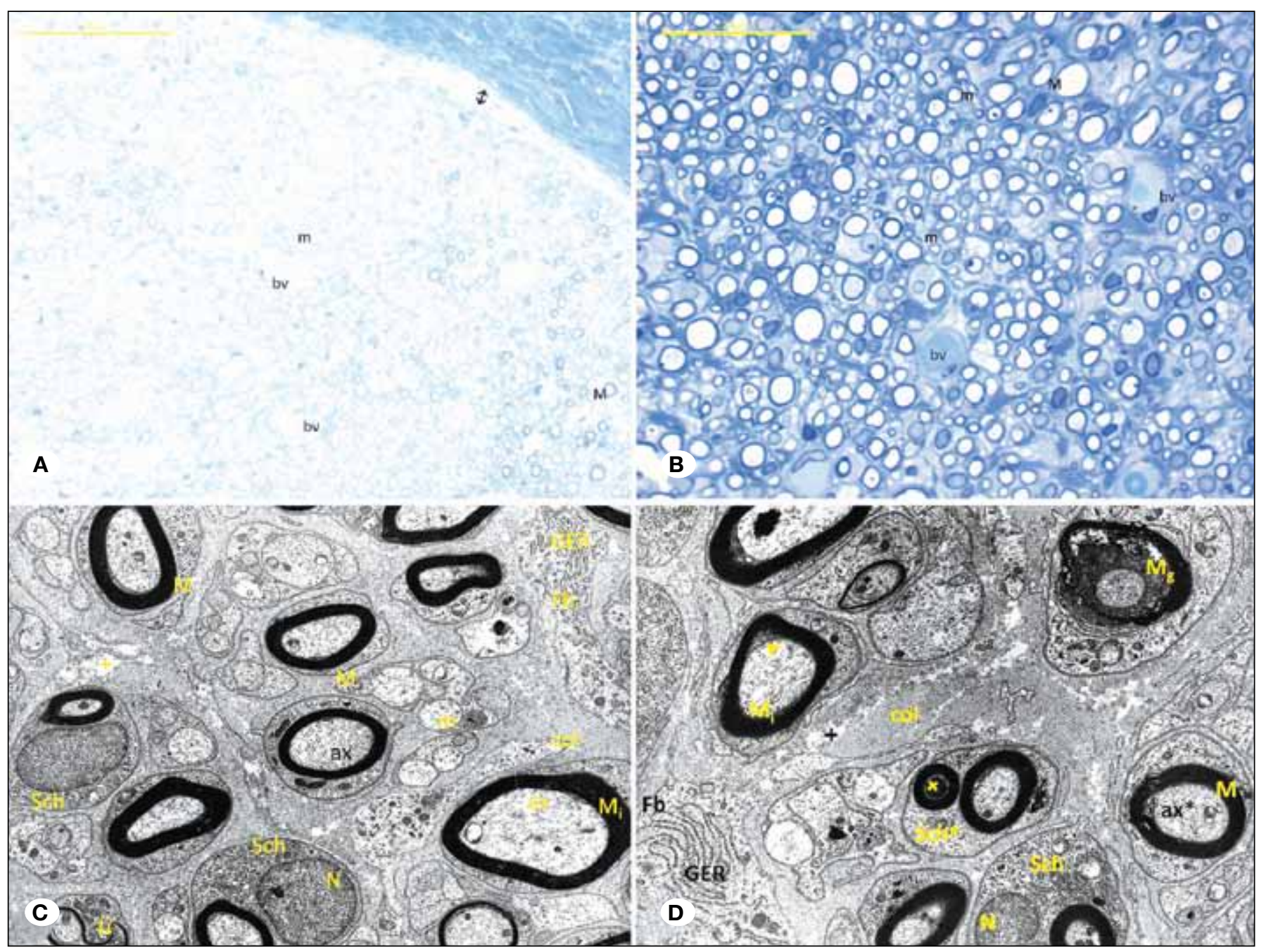

Figure 6: A, B) Trauma group +A-LA semi-thin section; M: Large diameter myelinated nerve fiber, M: Degenerative large diameter myelinated nerve fiber, m: small diameter nerve fiber with myelin, Bv: Blood vessels in endoneurial compartment, 2: connective tissue under epineurium (Toluidine Blue A x400-B x1000). C) Trauma+A-LA Group Thin Section; M: myelin sheath in normal configuration, Mi: myelin sheath in irregular thickness, m: Nerve fiber without myelin in normal structure, Sch: Schwann cell, N: Schwann cell nucleus, Fb: Fibroblast, GER: Granular endoplasmic reticulum +: edema in the intermediateconnective tissue, col: Collagen fibers (uranyl acetate\& lead citrate x3597). D) Trauma+ A-LA Group thin section; M: myelin sheath in normal configuration, Mi: myelin sheath with irregular thickness, ax: normal axon, Mg: Myelin sheath showing granular degeneration, ax*: Axon showing degenerative change, SchP: Polyaxonal Schwann cell, Sch: Schwann cell, +: edema in the intermediate connective tissue, col: collagen fibers, Fb: Fibroblast, GER: Granular endoplasmic reticulum (uranyl acetate\& lead citrate x4646).

It is known that target of peripheral nerve regeneration is the attainment of functional recovery. An important parameter confirming the functional improvement in our study was the g-ratio, which has been described as an index that reflects the optimal axonal myelination that leads to an appropriate conduction of action potentials (9). The g-ratio can simply be denoted as the ratio of axon circumference to myelin circumference, and this value will always be less than 1; however, remyelinated axons tend to have values closer to 1 than naturally myelinated axons. The g-ratio differences are less apparent in smaller axons (10). In this study, in the A-LA group, the percentage of myelinated fibers had optimal values (0.55-0.68) and similar results to the control group, indicating a stimulating effect of $A-L A$ on myelination.
While the antioxidant propery of A-LA is well known as reported in other previous studies report $(13,16)$, the presented study basically aimed to demonstrate the effects of long term use of A-LA and vitamin B12 histomorphologically and structurally.

Additionally, the histomorphometric analyses revealed that the A-LA and control groups had optimal $g$ values of myelinated nerve fibers. Similar $g$ values among the vitamin B12, saline and sham groups were observed, indicating a delayed or deficient myelination process. Ultrastructurally, vitamin B12 was found to be ineffective in restoring primarily functional parameters such as preventing trauma-induced edema, myelin sheath degeneration, polyaxonal Schwann cells, e-dense deposits in Schwann cell cytoplasm, structural changes, such 
as abnormal myelination and g-ratios. In contrast, A-LA was found to be more effective regarding sciatic nerve healing, including both structural and histomorphometric findings aspects, compared to vitamin B12 microscopically, shown as small and large diameter axons preserving the natural architecture in addition to large diameter axons with myelin showing intense structural damage. The axons showing remodelization of the myelin sheath in most of the areas were indicators of accelerated myelination process. However, in group V (vitamin B12 group), the number of myelinated nerve fibers significantly decreased at optimal intervals (0.55-0.68) being an indicator for poor nerve healing comparing to A-LA.

This study aimed the positive effect long-term use of A-LA on nerve healing and re-myelination in sciatic nerve after transection injuries with comparison of vitamin B12 as a dietary supplement. A-LA can be used in order to accelerate the nerve healing period after surgical repair because of the proven enhanced remodelization of the myelin sheath after injury with improved $\mathrm{g}$ ratios histomorphometrically. New medical treatment modalities should be developed for clinical use in peripheral nerve injuries.

\section{CONCLUSION}

A-LA administration following peripheral nerve transection injury is more effective in promoting nerve healing regarding the structural aspects of the sciatic nerve compared to vitamin B12 and also myelination of nerve fibers by increasing g-values. In clinical use, more experimental studies are necessary to emphasize the beneficial effects of the long-term use of these classes of drugs and vitamins to treat peripheral nerve transection injuries. Histomorphometric analyses and ultrastructural comparisons are necessary to increase the reliability of these studies.

\section{- REFERENCES}

1. Antoniadis G, Kretschmer T, Pedro MT, König RW, Heinen CP, Richter HP: latrogenic nerve injuries: Prevalence, diagnosis and treatment. Dtsch Arztebl Int 111:273-279, 2014

2. Bain JR, Mackinnon SE, Hunter DA: Functional evaluation of complete sciatic, peroneal, and posterior tibial nerve lesions in the rat. Plast Reconstr Surg 83: 129-138, 1989

3. Briani C, Dalla Torre C, Citton V, Manara R, Pompanin S, Binotto G, Adami F: Cobalamin deficiency: Clinical picture and radiological findings. Nutrients 5: 4521-4539, 2013

4. Chomiak T, Hu B: What is the optimal value of the g-ratio for myelinated fibers in the rat CNS? A theoretical approach. PLoS One 4: 7754, 2009

5. Corinne G, Mizisin LM, Austin N: B vitamins alleviate indices of neuropathic pain in diabetic rats. Eur J Pharmacol 612: 41-47,2009

6. Dinh P, Hazel A, Palispis W, Suryadevara S, Gupta R: Functional assessment after sciatic nerve injury in a rat model. Microsurgery 29: 644-649, 2009

7. Emmez H, Yildirim Z, Kale A, Tonge M, Durdag E, Borcek AO, Ucankus LN, Dogulu F, Kilic N, Baykaner MK: Anti-apoptotic and neuroprotective effects of a-lipoic acid on spinal cord ischemia-reperfusion injury in rabbits. Acta Neurochir (Wien) 152: $1591-1600,2010$
8. Erdi MF, Guney O, Kiyici A, Esen H: The effects of alpha lipoic acid on cerebral vasospasm following experimental subarachnoid hemorrhage in the rabbit. Turk Neurosurg 21: 527-533, 2011

9. Félix SP, Pereira Lopes FR, Marques SA, Martinez AM: Comparison between suture and fibrin glue on repair by direct coaptation or tubulization of injured mouse sciatic nerve. Microsurgery 33(6): 468-477,2013

10. Franklin RJ, Ffrench-Constant C: Remyelination in the CNS: From biology to therapy. Nat Rev Neurosci 9: 839-855, 2008

11. Hobbenaghi R, Javanbakh J, Hosseini E, Mohammadi $S$, Rajabian M, Moayeri P, Aghamohammad Hassan $M$ : Neuropathological and neuroprotective features of vitamin B12 on the dorsal spinal ganglion of rats after the experimental crush of sciatic nerve: An experimental study. Diagn Pathol $31: 123,2013$

12. Jacobs WB, Fehlings MG: The molecular basis of neural regeneration. Neurosurgery 53: 943-950,2003

13. Kaplan KA, Odabasoglu F, Halici Z, Halici M, Cadirci E, Atalay F, Aydin O, Cakir A: Alpha-lipoic acid protects against indomethacin-induced gastric oxidative toxicity by modulating antioxidant system. J Food Sci 77: 224-230, 2012

14. Kaplan T, Kafa IM, Cansev M, Bekar A, Karli N, Taskapilioglu $\mathrm{MO}$, Kanar F: Investigation of the dose-dependency of citicoline effects on nerve regeneration and functional recovery in a rat model of sciatic nerve injury. Turk Neurosurg 24: 52-62, 2014

15. Kurtoglu Z, Ozturk AH, Bagdatoglu C, Turac A, Camdeviren H, Uzmansel D, Aktekin M: Effects of trapedil on the sciatic nerve with crush injury: A light microscopic study. Neuroanatomy 3: 54-58,2004

16. Mitsui $Y$, Schmelzer JD, Zollman PJ, Mitsui M, Tritschler $\mathrm{HJ}$ : Low Alpha-lipoic acid provides neuroprotection from ischemia-reperfusion injury of peripheral nerve. J Neurol Sci 163: 11-16, 2009

17. Müller-Vahl H: Traumatic nerve damage: Causes, approaches and prognosis. Ann Neurol 45: 207-215, 1999

18. Roytta M, Salonen V: Long-term endoneurial changes after nerve transection. Acta Neuropathol 76(1):35-45, 1988

19. Saito F, Moore SA, Barresi R, Henry MD, Messing A, RossBarta SE, Cohn RD, Williamson RA, Sluka KA, Sherman DL, Brophy PJ, Schmelzer JD, Low PA, Wrabetz L, Feltri ML, Campbell KP: Unique role of dystroglycan in peripheral nerve myelination, nodal structure, and sodium channel stabilization. Neuron 38: 747-758, 2003

20. Scalabrino G: The multi-faceted basis of vitamin B12 (cobalamin) neurotrophism in adult central nervous system: Lessons learned from its deficiency. Prog Neurobiol 88: 203220, 2009

21. Senoglu M, Nacitarhan V, Kurutas EB, Senoglu N, Altun I, Atli Y, Ozbag D: Intraperitoneal Alpha-Lipoic Acid to prevent neural damage after crush injury to the rat sciatic nerve. $J$ Brachial Plex Peripher Nerve Inj 4:22, 2009

22. Sezer A, Guclu B, Kazanci B, Cakir M, Coban MK: Neuroprotective effects of agmatine in experimental peripheral nerve injury in rats: A prospective randomized and placebocontrolled trial. Turk Neurosurg 24(2):196-201,2014

23. Yuksel TN, Halici Z, Demir R, Cakir M, Calikoglu C, Ozdemir $G$, Unal D: Investigation of the effect of telmisartan on experimentally induced peripheral nerve injury in rats. Int $\mathrm{J}$ Neurosci 28:1-32, 2014 